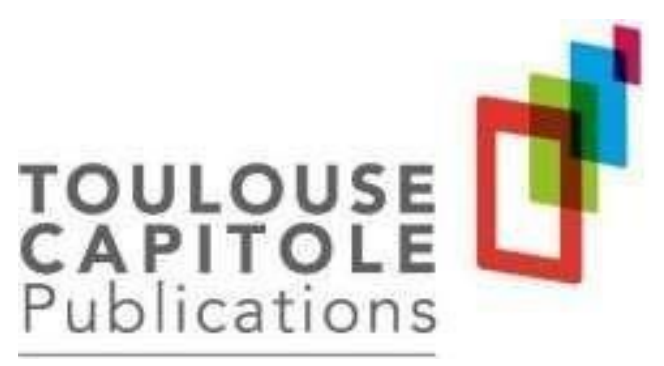

« Toulouse Capitole Publications » est l'archive institutionnelle de l'Université Toulouse 1 Capitole.

\title{
Les premières récusations au Conseil constitutionnel : réponses et nouveaux questionnements sur un instrument à double tranchant
}

\author{
PERLO NiCOLETTA
}

Référence de publication: PERLO (N.), « Les premières récusations au Conseil constitutionnel : réponses et nouveaux questionnements sur un instrument à double tranchant », Annuaire international de justice constitutionnelle, $\mathrm{n}^{\circ}$ XXVII, p. 61, 2011. 


\section{Les premières récusations au Conseil constitutionnel : réponses et nouveaux questionnements sur un instrument à double tranchant}

Depuis la révision constitutionnelle du 23 juillet 2008, le Conseil constitutionnel est en train de vivre de formidables transformations. L'introduction de la question prioritaire de constitutionnalité (QPC) a accéléré le processus de juridictionnalisation des fonctions du Conseil, qui devient une véritable Cour, à l'instar des autres organes européens de contrôle de la constitutionnalité de la loi. Se conformant à la jurisprudence de la Cour européenne des droits de l'homme $(\mathrm{CEDH})^{1}$ le contentieux constitutionnel $a$ posteriori français est désormais soumis aux règles du procès équitable, affirmées par l'article $6 \S 1$ de la Convention européenne des droits de l'homme. Par conséquent, le Conseil constitutionnel doit assurer un déroulement du procès transparent, permettant aux acteurs du contentieux de bénéficier d'une procédure contradictoire et au juge constitutionnel d'apporter à sa décision des garanties d'impartialité ${ }^{2}$.

Pour ce qui est de l'impartialité, l'article 3 de l'ordonnance du 7 novembre 1958 faisait déjà référence à l'obligation pesant sur les membres du Conseil constitutionnel d'exercer leurs fonctions «en toute impartialité ». L'impartialité du juge est, en effet, un principe incontournable non seulement pour les juridictions ordinaires, auxquelles il est traditionnellement rattaché ${ }^{3}$, mais aussi pour les juridictions de la constitutionnalité de la loi ${ }^{4}$. La garantie de l'équilibre constitutionnel d'une société pluraliste, au travers de la «résolution judiciaire des plus importants conflits politiques par des normes juridiques constitutionnelles positives ${ }^{5}$, impose en effet que le juge de la Constitution soit indépendant et impartial, afin que les parties en conflit reconnaissent son autorité comme légitime et se soumettent à ses décisions. Si l'indépendance de l'organe «s'exprime en externe, par rapport à d'autres pouvoirs que le pouvoir judiciaire, celui de l'exécutif et du législatif » ${ }^{6}$, en revanche, l'impartialité est «davantage liée à l'organisation et au fonctionnement internes des juridictions, aux qualités personnelles du juge ${ }^{7}$. Depuis l'arrêt Piersack c/ Belgique ${ }^{8}$, la Cour européenne a opéré une distinction nette entre l'impartialité subjective, qui tient «au juge en tant qu'homme », et l'impartialité objective, qui est liée à l'organe ou à la fonction ${ }^{9}$. Les notions d'indépendance et d'impartialité sont liées par un rapport de causalité : «l'indépendance est un préalable à l'impartialité ; on ne peut être impartial, si, déjà, on n'est pas indépendant ; mais à l'inverse, un juge indépendant de tout pouvoir peut devenir partial dans un dossier particulier $\gg 10$.

Si sur la base de différents critères ${ }^{11}$, le Conseil constitutionnel français apparaît comme une juridiction indépendante, jusqu'en 2010, aucun dispositif législatif, mise à part l'abstention volontaire des juges, ne permettait de garantir son impartialité. Toutefois, après la révision constitutionnelle de 2008 , le règlement intérieur sur la procédure suivie devant le Conseil constitutionnel pour les questions prioritaires de constitutionnalité du 4 février $2010^{12}$, tirant les conséquences des nouvelles compétences juridictionnelles du Conseil, a institutionnalisé la pratique du déport et a introduit la possibilité de récuser les juges constitutionnels.

La France rejoint ainsi de nombreux pays européens qui, bien avant l'extension des principes du procès équitable au contentieux constitutionnel, avaient prévu le déport et la récusation des membres de leurs cours constitutionnelles. Notamment, les législateurs allemand ${ }^{13}$ et belge ${ }^{14}$ ont conçu un régime spécifique pour les juges constitutionnels. Les droits espagnol ${ }^{15}$ et portugais ${ }^{16}$, en revanche, ont renvoyé aux dispositions relatives aux juridictions ordinaires ${ }^{17}$. 
Toutefois, par rapport aux systèmes étrangers, le déport et la récusation des juges constitutionnels français présentent des caractéristiques tout à fait originales, qui dévoilent une certaine méfiance de l'organe visà-vis des deux instruments, perçus plus comme une menace pour la légitimité des juges que comme une véritable garantie d'impartialité. Les deux mécanismes ne sont pas réglementés par des lois organiques ou ordinaires. Le Conseil constitutionnel, exerçant son pouvoir réglementaire ${ }^{18}$, a élaboré un régime adapté à ses propres exigences, donnant aux deux dispositifs un cadre procédural souple, qui laisse une marge importante à la pratique et qui limite fortement les risques d'atteinte au fonctionnement de l'organe.

Depuis l'entrée en vigueur du règlement intérieur, plusieurs juges constitutionnels se sont déportés, dans la continuité avec la pratique des abstentions volontaires, ratifiée par le Conseil ${ }^{19}$. En revanche, l'exercice du nouveau droit de récusation était attendu avec quelques craintes ${ }^{20}$. La récusation peut être une arme à double tranchant. D'une part, elle «réalise l'impartialité attendue d'un juge ${ }^{21}$ : si les parties n'exercent pas leur droit de récusation, cela signifie qu'elles reconnaissent la pleine impartialité et donc la pleine légitimité de la décision rendue ${ }^{22}$. D'autre part, la récusation jette inévitablement une ombre sur le juge qui en fait l'objet, mettant en doute sa capacité d'adopter une décision équitable. Les premières demandes de récusation ont été posées le 17 mai 2011, contre six des onze membres du Conseil par Arnaud Montebourg, député socialiste et président du conseil général de Saône-et-Loire ${ }^{23}$. L'analyse des demandes de récusation soulevées et de la décision adoptée par le Conseil constitutionnel apparaît comme un instrument fort utile pour évaluer concrètement le fonctionnement et les effets de la récusation française. Tout d'abord, en l'absence de toute indication réglementaire, ce premier cas permet de déceler quelques règles de principe déterminant les causes de récusation invocables par les parties (I). En second lieu, la première application de la récusation précise les règles de sa procédure, laissée plutôt indéfinie par le règlement intérieur (II).

\section{Les causes de récusation}

Le règlement ne contient pas une liste de causes de récusation. Contrairement aux droits espagnol ${ }^{24}$ et belge ${ }^{25}$ qui renvoient aux causes d'abstention ou de récusation prévues pour les juridictions ordinaires, ou encore au droit allemand, qui énumère des causes de récusation spécifiques pour l'organe de contrôle constitutionnel $^{26}$, le Conseil a préféré laisser indéterminé le champ d'application de la récusation de ses membres.

En cela, les Sages se conforment à la jurisprudence de la Cour de cassation qui, en 1998, a considéré que l'article 341 du nouveau Code de procédure civile, dressant une liste des causes de récusation, «n'épuise pas nécessairement l'exigence d'impartialité requise de toute juridiction ${ }^{27}$. Pour la Cour de cassation, l'article $6 \S 1$ de la Convention européenne est directement invocable devant les juridictions nationales et, par conséquent, omet la limitation formelle des causes de récusation de l'article $341^{28}$.

Le Conseil s'inscrit dans cette jurisprudence, tout en posant, cependant, une limite importante aux motifs de récusation : «Le seul fait qu'un membre du Conseil constitutionnel a participé à l'élaboration de la disposition législative faisant l'objet de la question de constitutionnalité ne constitue pas en lui-même une cause de récusation $\gg^{29}$.

Étant donnée la composition du Conseil constitutionnel, la limite tend à éviter le risque de blocage de l'institution mais, selon certains, a «vidé de sa substance l'hypothèse de récuser un juge constitutionnel » 
30 . Les premières demandes de récusation relèvent immédiatement cette contradiction. Un conflit se déclenche ainsi entre le requérant et le Conseil constitutionnel autour de la légitimité de la cause d'exclusion contenue dans l'article 4 du règlement intérieur (A). Une analyse attentive de la demande de récusation et de la décision du Conseil permet ensuite d'esquisser les premières règles jurisprudentielles déterminant les causes de récusations admissibles (B).

\section{A. Le conflit sur la légitimité de la cause d'exclusion de la récusation}

M. Montebourg fonde l'argument de l'illégitimité de la limite posée par l'article 4 du règlement intérieur sur l'arrêt McGonnell cl Royaume-Uni de la Cour européenne des droits de l'homme ${ }^{31}$. En revanche, le secrétaire général du Conseil justifie la légitimité de cette limite sur la base d'un autre arrêt de la Cour de Strasbourg, Morel c/France, dont les conclusions sont apparemment opposées à l'arrêt McGonnell (1). Un conflit se déclenche, ouvrant des questionnements ultérieurs, qui touchent notamment à la composition du Conseil et aux critères de nomination de ses membres (2).

\section{1) Le conflit entre le requérant et le Conseil constitutionnel}

Le secrétaire général du Conseil constitutionnel, dans son commentaire à la procédure pour les questions prioritaires de constitutionnalité ${ }^{32}$, justifie la légitimité de la cause d'exclusion de la récusation contenue dans l'article $4 \mathrm{du}$ règlement sur le fondement de l'arrêt Morel c/France ${ }^{33}$ de la Cour européenne. Cet arrêt concerne un cas d'exercice cumulatif de fonctions distinctes par un juge en matière civile. Notamment, lors d'une procédure de redressement judiciaire, le juge- commissaire avait fait également partie de la formation de jugement en qualité de président de la chambre. La double fonction recouverte par la même personne dans le cadre de la même affaire a soulevé le doute des parties sur l'impartialité de la décision du tribunal.

La Cour de Strasbourg statue que : «Le simple fait, pour un juge, d'avoir déjà pris des décisions avant le procès ne peut passer pour justifier en soi des appréhensions relativement à son impartialité. Ce qui compte est l'étendue des mesures adoptées par le juge avant le procès. De même, la connaissance approfondie du dossier par le juge n'implique pas un préjugé empêchant de le considérer comme impartial au moment du jugement sur le fond. Enfin, l'appréciation préliminaire des données disponibles ne saurait non plus passer comme préjugeant l'appréciation finale. Il importe que cette appréciation intervienne avec le jugement et s'appuie sur les éléments produits et débattus à l'audience» ${ }^{34}$.

Le secrétaire général du Conseil, appliquant la décision Morel à la juridiction constitutionnelle, arrive aux conclusions suivantes : «Cette jurisprudence tend à retenir que le seul fait d'avoir participé à l'élaboration de la norme contestée ne constitue pas en soi une cause de récusation. Il ne devrait en aller autrement que si les actes accomplis impliquent que leur auteur a porté une appréciation sur la constitutionnalité de cette norme $\gg 35$.

Ainsi, le simple fait de participer à l'élaboration de la norme contestée est apparenté «au simple fait pour un juge d'avoir déjà pris des décisions avant le procès » ${ }^{36}$. Pour que le doute sur l'impartialité d'un juge soit admissible, il faut que ce dernier ait exprimé, dans le passé, de façon implicite ou explicite, une appréciation sur la constitutionnalité de la norme.

Le requérant, M. Montebourg s'appuie en revanche sur l'arrêt McGonnell c/ Royaume-Uni ${ }^{37}$ de la Cour 
de Strasbourg et sur l'adage anglais, fondateur de la théorie de l'apparence, «Justice must not only be done, it must be seen to be done ${ }^{38}$. Cet arrêt concerne un cas de partialité d'un juge dans l'exercice successif et cumulatif de fonctions législatives et de fonctions juridictionnelles. L'affaire est relative au bailli, le premier citoyen et premier représentant de l'île de Guernesey, qui préside les States of Deliberation, organe législatif, et il est le premier magistrat de la Royal Court, organe judiciaire. Dans le cas d'espèce, le bailli avait présidé les States of Deliberation lors de l'adoption de l'acte en cause dans l'affaire postérieure du requérant. Sur cette affaire avait ensuite statué la Royal Court, dans laquelle siégeait également le bailli en tant que juge ${ }^{39}$. Le bailli s'est donc trouvé à appliquer, et par conséquent à interpréter, des dispositions tout en ayant participé à leur élaboration. Cet exercice successif et cumulatif de fonctions législatives et juridictionnelles est jugé négativement par la Cour européenne, qui estime que : «Toute participation directe à l'adoption de textes législatifs ou réglementaires peut suffire à jeter le doute sur l'impartialité judiciaire d'une personne amenée ultérieurement à trancher un différend sur le point de savoir s'il existe des motifs justifiant que l'on s'écarte du libellé des textes législatifs ou réglementaires en question ${ }^{40}$.

La Cour tient également à préciser que le fait pour le bailli de présider les States of Deliberation ne peut pas amener à considérer que ce dernier remplisse un simple rôle protocolaire, sans exercice de fonctions législatives actives. Pour la Cour «même un rôle constitutionnel purement protocolaire est à considérer comme une "fonction" » ${ }^{41}$.

La décision McGonnell confirme la jurisprudence Procola c/Luxembourg de septembre 1995, à laquelle la Cour ne manque pas de se référer ${ }^{42}$. Dans ce cas, la Cour européenne avait condamné le système luxembourgeois. La condition d'impartialité n'était pas respectée puisque la double appartenance d'un membre du Conseil d'État à la section administrative et à la section du contentieux créait le risque que les mêmes personnes puissent connaître successivement d'une affaire en tant que consultants juridiques du Gouvernement et en tant que juges administratifs ${ }^{43}$.

La mise en confrontation des jurisprudences McGonnell et Morel conduit à s'interroger sur la décision la plus adaptée au contentieux constitutionnel et à réfléchir sur la compatibilité de la garantie de l'impartialité avec la préservation du bon fonctionnement du Conseil constitutionnel.

\section{2) L'équilibre difficile entre la garantie de l'impartialité et le bon fonctionnement de l'organe constitutionnel}

L'arrêt Morel constitue sans doute la jurisprudence de référence quand il s'agit d'apprécier la «partialité dans l'exercice successif et cumulatif, pour la même affaire, de fonctions judiciaires distinctes au sein de l'organe exerçant la fonction juridictionnelle ${ }^{44}$. En revanche, l'appréciation de l'impartialité du juge constitutionnel semble mieux s'encadrer dans le cas de figure d'un juge qui exerce successivement et cumulativement des fonctions législatives et des fonctions juridictionnelles ${ }^{45}$, comme dans les affaires McGonnell et Procola. Le Conseil constitutionnel français est notamment composé d'anciens parlementaires et membres de l'Exécutif. Par conséquent, la Haute juridiction peut avoir à connaître de la compatibilité à la Constitution de textes que certains de ses membres ont pu défendre ou combattre au moment de leur adoption par le Parlement. Il ne s'agit donc pas de l'exercice cumulatif de fonctions juridictionnelles, comme dans l'affaire Morel, mais de l'exercice cumulatif de fonctions législatives et juridictionnelles. 
Si la position défendue par le secrétaire général du Conseil constitutionnel est alors contestable d'un point de vue strictement juridique, son but est toutefois compréhensible. Le Conseil tend en effet à préserver son fonctionnement. Puisque cet organe est traditionnellement composé par des personnes qui ont recouvert par le passé des charges politiques et institutionnelles, il est fort probable que les juges aient déjà connu dans l'exercice de leurs précédentes fonctions législatives ou administratives les dispositions soumises au contrôle de constitutionnalité a posteriori. La solution adoptée par le règlement est donc celle d'évincer la cause de récusation qui le plus souvent pourrait être avancée : l'exercice successif et cumulatif de fonctions législatives et judiciaires pour le même texte législatif.

D'ailleurs, cette solution n'est pas qu'une prérogative française. L'alinéa 2 de l'article 101 de la loi spéciale sur la Cour constitutionnelle belge, composée par moitié d'anciens parlementaires ${ }^{46}$, précise également que la participation à l'élaboration de la loi faisant l'objet du recours «ne constitue pas en soi une cause de récusation ${ }^{47}$. En Allemagne, le paragraphe 18 de la loi du 12 mars 1951 sur la Cour constitutionnelle allemande, composée de personnalités issues du monde politique et universitaire, indique que la «participation à une procédure législative » ou «l'expression d'une opinion scientifique concernant une question juridique pouvant être significative pour la procédure » ne constituent pas des causes de récusation valables.

La solution adoptée par les différentes cours constitutionnelles entre toutefois en conflit avec la pleine garantie du principe d'impartialité de la juridiction constitutionnelle. Comme les jurisprudences McGonnell et Procola l'indiquent, le fait d'avoir participé en précédence à l'élaboration d'une disposition législative peut à juste titre faire douter que le juge constitutionnel ait déjà un préjugé sur la constitutionnalité de la norme et qu'il sera tenté de le suivre, portant atteinte à l'impartialité du jugement. Face à ce conflit entre, d'une part, la préservation du fonctionnement du Conseil et, de l'autre, la sauvegarde de l'impartialité, faudrait-t-il en déduire que la garantie de l'impartialité de la juridiction constitutionnelle est incompatible avec la structure même de l'organe et sa mission ?

Dans le cas français, le conflit pourrait être partiellement résolu réformant la composition du Conseil constitutionnel. Cela ouvre inévitablement le débat sur les critères de nomination des Sages. La composition du Conseil est en effet fonctionnelle à la mission originaire pour laquelle il a été institué en 1958 : «une arme contre la déviation du régime parlementaire » ${ }^{48}$. Malgré les importantes évolutions du rôle et des fonctions du Conseil et les nombreuses propositions de réforme ${ }^{49}$, les trois autorités politiques de nomination ne sont toujours pas liées dans leur choix par des critères permettant de tempérer le caractère politique de la nomination et de renforcer la compétence technico-juridique de l'organe. Tout en sachant que le caractère politique de la juridiction constitutionnelle est intrinsèque à sa nature et au lien étroit qui existe entre la Constitution et la politique ${ }^{50}$, l'introduction de critères capables de renforcer les compétences technico-juridiques de l'institution faciliterait l'équilibre entre la garantie de l'impartialité et le bon fonctionnement du Conseil. Un juge fort des compétences techniques qui ont permis sa nomination est certainement plus indépendant de son autorité de nomination et moins susceptible d'être récusé qu'un juge qui a été fortement impliqué dans la vie parlementaire et qui a été nommé davantage en vertu de sa sensibilité politique ${ }^{51}$. Cela ne signifie pas prôner une technicisation de l'organe. Les juristes peuvent avoir en effet une sensibilité politique très poussée, maîtrisée et tempérée, toutefois, par la rigueur de la discipline du droit.

\section{B. Vers la création de règles prétoriennes définissant les causes de récusation}


M. Montebourg, considérant que la limite à la récusation prévue par l'article 4 du règlement intérieur ne garantit pas le plein respect du principe d'impartialité des juges constitutionnels, invoque l'applicabilité directe de l'article $6 \$ 1$ de la Convention EDH au droit interne ${ }^{52}$. Il sollicite ainsi la récusation de six juges du Conseil : M. le Président Debré, M. Charasse, M. Barrot, M. Haenel, M. Chirac et M. Steinmetz 53 . Les raisons de la récusation vont de toute évidence à l'encontre de l'article 4, mais elles pourraient être considérées conformes à la jurisprudence de la Cour de Strasbourg (1). En revanche, le Conseil constitutionnel donne une interprétation stricte de l'article 4 du règlement intérieur. Malgré l'absence de la motivation du délibéré, les premières règles prétoriennes du Conseil sur les causes de récusation commencent à se préciser (2).

\section{1) Les causes de récusation alléguées par le requérant}

Le président du département Saône-et-Loire demande tout d'abord la récusation de M. le Président du Conseil, Jean-Louis Debré, puisqu'à l'époque de l'approbation des lois faisant l'objet du contrôle, il était président de l'Assemblée nationale et «a de ce fait présidé les débats ayant conduit au vote de ces textes » 54 . Puisque dans l'arrêt McGonnell cité par le requérant la Cour européenne a précisé que «même un rôle constitutionnel purement protocolaire est à considérer comme une "fonction" $\gg{ }^{55}$, la présidence des débats parlementaires à l'Assemblée nationale pourrait être considérée comme une participation active à l'élaboration de la disposition législative faisant l'objet du contrôle de constitutionnalité a posteriori.

L'application directe de l'article $6 \$ 1$ de la Convention EDH pourrait donc légitimer la récusation du Président.

M. Montebourg exerce ensuite son droit de récusation à l'encontre de M. Jacques Chirac qui, à l'époque de l'adoption des dispositions législatives, était Président de la République et qui, de ce fait, aurait «initié, conformément au rôle que la pratique institutionnelle a confié au chef de l'État sous la Ve République, lesdits textes ${ }^{56}$. De toute évidence, le président de la République joue un rôle actif dans la procédure d'élaboration de la loi. Suite à l'introduction de l'élection au suffrage direct du président ${ }^{57}$ et à la réduction du mandat présidentiel à cinq ans ${ }^{58}$, la pratique institutionnelle a sensiblement étendu les pouvoirs de ce dernier. Désormais, loin du rôle d'arbitre qui lui est conféré par le texte constitutionnel ${ }^{59}$, le président de la République «est au premier plan du jeu politique ». «Lorsqu'il est le pivot de la majorité parlementaire, il est le maître du processus législatif et [...] lorsque, par exception, il ne peut plus l'être (cohabitation), il est sinon le chef de l'opposition au gouvernement, du moins une force très active de celle-ci » ${ }^{60}$. L'impartialité de M. Jacques Chirac dans le contrôle de constitutionnalité a posteriori de lois adoptées pendant son mandat présidentiel semble donc pouvoir être mise en doute à juste titre ${ }^{61}$.

Trois autres juges sont récusés puisque, en tant que parlementaires, ils ont participé au vote des lois faisant l'objet de la QPC. Il s'agit de Michel Charasse et d'Hubert Haenel, qui à l'époque étaient sénateurs, et de Jacques Barrot, qui était député et président du groupe UMP à l'Assemblée nationale. Selon la jurisprudence de la CEDH McGonnell et Procola, et contrairement à l'article 4 du règlement intérieur, le vote en faveur ou contre une loi peut être considéré comme une forme de participation active à l'élaboration de la disposition législative, indépendamment du degré d'implication du parlementaire dans l'adoption de la norme.

En effet, si nous considérons, comme le secrétaire général du Conseil constitutionnel d'ailleurs le fait ${ }^{62}$, que le doute sur l'impartialité d'un juge peut surgir lorsque ce dernier a accompli précédemment des actes 
exprimant un jugement sur la constitutionnalité de la loi, le vote pourrait constituer un acte de telle sorte. D'un point de vue juridico-constitutionnel, il paraît paradoxal qu'un parlementaire vote en faveur d'une disposition législative tout en étant conscient qu'elle est contraire aux principes affirmés par le bloc constitutionnel. Par conséquent, dans un «État de droit constitutionnel ${ }^{63}$, si un parlementaire vote en faveur d'une loi, cela implique qu'il la considère comme manifestement conforme à la Constitution. En revanche, si un parlementaire vote contre un projet de loi, il se peut qu'il ne soit pas d'accord avec le contenu de la norme, mais qu'il la considère tout à fait conforme à la norme suprême. Dans le cas contraire, s'il considère que le projet de loi porte atteinte au bloc constitutionnel, il pourra agir en saisissant le Conseil constitutionnel avec cinquante-neuf autres sénateurs ou députés et déclencher ainsi le contrôle $a$ priori. Dans le cas d'espèce, Messieurs Charasse, Barrot et Haenel ont tous voté en faveur des lois du 18 décembre et du 30 décembre 2003 manifestant implicitement que les normes en question étaient conformes à la Constitution.

La récusation visant Pierre Steinmetz paraît plus discutable. Selon M. Montebourg la fonction de directeur du cabinet du premier ministre occupée par M. Steinmetz en 2003 impliquerait ipso facto sa participation à l'élaboration des textes de loi ${ }^{64}$. Cet argument semble difficilement fondé au vu de la jurisprudence McGonnell et Procola de la Cour de Strasbourg. Le doute sur l'impartialité d'un juge est légitime si basé sur des actes accomplis en précédence par ce dernier, pouvant induire les parties du contentieux à penser que, dans la décision, le juge est influencé par des préjugés élaborés auparavant. Ainsi, la participation au vote en faveur ou contre une loi, la saisine du Conseil constitutionnel pour déclencher un contrôle de constitutionnalité a priori, la proposition d'une loi ou bien d'amendements sont des comportements qui peuvent faire douter de l'impartialité du juge constitutionnel. De façon explicite ou implicite, le juge a en effet déjà pris position sur la conformité de la disposition à la Constitution. En revanche, le directeur du cabinet du Premier ministre est un haut fonctionnaire qui, tout en ayant connaissance des projets de lois débattus au Parlement, ne joue aucun rôle institutionnel dans la procédure législative.

\section{2) Les règles interprétatives esquissées par le Conseil en matière de récusation}

La lettre de réponse envoyée au requérant ${ }^{65}$ se limite à communiquer la décision adoptée par le Conseil «suite à la demande de récusation présentée », sans motiver en détail les raisons du délibéré final ${ }^{66}$.

De façon pour le moins ambiguë, le secrétaire général annonce que «Messieurs Jacques Barrot et Michel Charasse ne participeront pas à la décision du Conseil constitutionnel sur les questions prioritaires de constitutionnalité n 2011-142 à 2011-145 ». La formule utilisée ne permet pas de comprendre si les deux juges ont décidé d'acquiescer à la demande de récusation de M. Montebourg pour les QPC n 2011-142 et 2011-143, ou bien s'ils avaient déjà décidé de se déporter indépendamment de la requête. La lecture des commentaires des décisions aux Nouveaux cahiers du Conseil constitutionnel fait pencher pour la seconde option. En effet, annonçant que le Conseil a été saisi pour la première fois d'une demande de récusation de certains de ses membres, les Cahiers affirment: «[...] Outre MM. Barrot et Charasse, qui ne siégeaient pas dans ces QPC, M. Chirac ne participe plus aux travaux du Conseil constitutionnel depuis mars 2011. Par ailleurs, le Conseil a repoussé les demandes de récusation concernant MM. Debré, Haenel et Steinmetz $[\ldots] \gg 67$.

Par conséquent, Messieurs Barrot et Charasse n'ont pas refusé la demande de récusation, puisqu'autrement elle aurait fait l'objet du contrôle du Conseil comme pour les autres juges visés, en conformité de l'article 4 du règlement. Les deux parlementaires auraient donc décidé de s'abstenir avant même l'enregistrement 
de la demande de récusation au secrétariat général.

En l'absence de toute motivation, la compréhension des raisons de l'acquiescence à la demande de récusation ou de l'abstention volontaire des deux juges est difficile. Toutefois, malgré le fait que les déports ne soient pas motivés non plus ${ }^{68}$, un regard sur les abstentions pratiquées par les deux juges dans le cadre de précédentes QPC peut aider à expliquer leur absence dans le cas d'espèce et à fournir ainsi des pistes pour l'établissement d'une liste de causes de récusation admissibles. Dans le passé, M. Charasse s'est déporté lors de décisions concernant la décentralisation, en raison, comme présumé par Emmanuel Cartier, de son implication politique dans ce domaine, en tant qu'ancien élu local, sénateur et membre du Comité des finances locales ${ }^{69}$. À ces titres, il a en effet défendu de façon manifeste et publique l'intérêt financier des collectivités territoriales ${ }^{70}$. Quant à M. Jacques Barrot, la raison de son absence pourrait être liée à son implication dans la politique des collectivités territoriales en tant que président du comité départemental de la fédération UMP de la Haute-Loire et président du conseil général de la Haute-Loire en 2003.

Dans les deux cas, le risque de partialité est lié plus à des raisons subjectives qu'objectives. L'absence des deux parlementaires ne semble pas s'attacher à leur participation au vote en faveur des dispositions législatives visées, comme M. Montebourg argumente dans sa demande. C'est plutôt l'implication manifeste des deux juges dans la politique des collectivités territoriales qui peut être ressentie comme une atteinte possible à leur impartialité. Preuve en est que la demande de récusation du sénateur Hubert Haenel, sur le fondement de sa participation au vote, a été rejetée par le Conseil sur la base de la cause d'exclusion de l'article 4.

Le vote en faveur d'une loi est-il donc une cause de récusation admissible pour le Conseil ? D'une manière générale, une lecture stricte de l'article 4 semble exclure cette hypothèse, qui rentrerait dans la limite du quatrième alinéa. Toutefois, la pratique du déport indique que si le simple vote d'une loi ne peut constituer en lui seul une cause d'abstention, en revanche la proposition d'une loi ou d'un amendement manifestent un degré d'implication qui peut faire douter ensuite de l'impartialité du parlementaire devenu juge

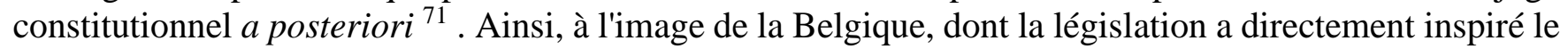
règlement intérieur français ${ }^{72}$, «lorsqu'un des juges a été, en tant que parlementaire, directement à l'origine d'une disposition soumise au contrôle de la Cour, soit comme auteur de la proposition, soit comme auteur d'un amendement à la proposition » ${ }^{73}$, il peut être récusé par les parties au cas où il ne se déporte pas volontairement.

Le rejet par le Conseil de la demande de récusation de M. Debré fournit des éléments ultérieurs pour une réflexion sur les causes de récusation admissibles. Les Sages n'ont pas considéré que la présidence des travaux de l'Assemblée nationale lors de la discussion de la norme faisant l'objet du contrôle constitue un motif légitime pour suspecter de l'impartialité du juge. Il s'agit pour le Conseil soit une forme de participation à l'élaboration de la norme rentrant dans la cause d'exclusion de l'article 4, soit, en dépit de la jurisprudence McGonnell de la Cour européenne, une activité sans aucune influence sur l'adoption de la loi.

De même, concernant la demande de récusation de M. Steinmetz, la direction du cabinet du premier ministre dans la période d'adoption de la norme n'a pas été considérée par le Conseil comme un risque objectif d'atteinte à l'impartialité du juge. La décision était prévisible, puisque cette cause de récusation était manifestement infondée au vu de l'article 4 du règlement et difficilement justifiable à la lumière de 
la jurisprudence européenne en la matière.

Enfin, concernant l'ex président de la République Jacques Chirac, M. Guillaume évite de répondre sur le point de savoir si Monsieur Chirac pourrait effectivement être suspect de partialité en raison du rôle de président de la République revêtu lors de l'adoption des dispositions en question. Le secrétaire général affirme tout simplement que : «Monsieur Jacques Chirac ne participe pas aux travaux du Conseil constitutionnel depuis mars $2011 »$.

En conclusion, la première décision du Conseil sur une demande de récusation donne une interprétation stricte de l'article 4 du règlement intérieur. Le simple vote de la loi, la présidence des débats parlementaires à l'Assemblée nationale et la direction du cabinet du premier ministre lors de l'adoption de la disposition législative sont considérés comme des formes de «participation à l'élaboration de la disposition législative » ne pouvant pas valoir comme causes de récusation. En revanche, la pratique du déport semble indiquer qu'une implication plus active du juge dans l'adoption de la loi pourrait créer des suspects sur son impartialité et légitimer par conséquent une demande de récusation à son encontre.

\section{La procédure de récusation}

La première demande de récusation permet d'observer les modalités d'application de l'article 4 du règlement intérieur. De nombreuses interrogations se posaient, étant donné que le régime élaboré par le Conseil laisse une marge importante à la pratique procédurale. Si la procédure de la demande de récusation est bien définie et n'a pas réservé de surprises (A), la procédure de la décision du Conseil, en grande partie remise à des choix discrétionnaires de l'institution, a été précisée par sa première application (B).

\section{A. La procédure de la demande de récusation}

La première demande de récusation a été respectueuse des principales règles formelles exigées par l'article 4 du règlement intérieur (1). Toutefois, puisqu'elle a concerné six juges, elle n'a pas manqué de poser la question de la compatibilité du quorum fixé pour la délibération du Conseil avec la garantie de l'impartialité des juges (2).

\section{1) La forme de la demande de récusation}

Le deuxième alinéa de l'article 4 du règlement intérieur de 2010 reconnaît à toute partie ou à son représentant, muni à cette fin d'un pouvoir spécial, le droit de demander la récusation d'un membre du Conseil constitutionnel. Les «parties » du contentieux constitutionnel a posteriori sont le demandeur, c'est-à-dire le justiciable, et le ministère public en matière pénale ou civile, ainsi que le Gouvernement, en charge de défendre la disposition législative. Puisque la récusation est un droit personnel, la partie ellemême doit l'exercer ou bien donner un pouvoir spécial à son avocat pour qu'il le fasse à sa place. Dans le cas d'espèce, la demande de récusation a été présentée par le département de Saône-et-Loire, représenté directement par son président, M. Arnaud Montebourg.

La demande a été enregistrée auprès du secrétariat général du Conseil dans le respect du délai et de la forme prévus par le règlement intérieur. Notamment, elle a été enregistrée «avant la date fixée pour la réception des premières observations », qui est établie par le secrétaire général sous forme d'un avis, suite 
à la notification de la question préjudicielle de constitutionnalité ${ }^{74}$. Comme dans d'autres cas $^{75}$, le règlement ne précise pas le délai, mais laisse le Conseil libre de se déterminer au cas par cas, en fonction de l'évolution de la procédure ${ }^{76}$. À l'heure actuelle, le délai pour présenter les premières observations est fixé à environ vingt jours ${ }^{77}$.

La demande de récusation de M. Montebourg était contenue dans un «écrit spécialement motivé » ${ }^{78}$, tendant à prouver l'existence des causes de récusation présentées. L'impartialité des juges constitutionnels, en effet, «se présume jusqu'à preuve du contraire ${ }^{79}$. Il faut donc que les requérants apportent des éléments objectifs pour prouver la partialité du juge.

\section{2) Le respect du quorum fixé pour la délibération du Conseil}

La demande du département de Saône-et-Loire vise six juges du Conseil. Cela ne semble pas contredire le règlement. S'il est vrai que l'article 4 se réfère à la récusation «d'un membre du Conseil », il ne faut pas donner une interprétation littérale de cette expression. Il semblerait, en effet, contraire à une garantie réelle d'impartialité que le droit de récusation des parties ne puisse s'exercer qu'à l'encontre d'un seul juge, alors que, dans la même affaire, d'autres membres seraient suspects de ne pas pouvoir juger de façon impartiale.

Toutefois, cela se heurte à la règle générale selon laquelle le Conseil pour statuer valablement nécessite le vote d'au moins sept membres. Pour garantir le principe d'impartialité, il serait possible de considérer la récusation comme un «cas de force majeure », en la faisant rentrer dans le cadre de l'exception prévue par l'article 14 de l'ordonnance du 7 novembre 1958. Il faudrait alors adopter une interprétation souple de la force majeure, excluant notamment l'imprévisibilité ${ }^{80}$. Le cas d'exception serait d'ailleurs justifié par le fait que le blocage du Conseil «déboucherait sur un déni de justice qui, en soi, parce qu'il serait la négation même du droit au juge, justifie que la règle soit écartée ${ }^{81}$.

En revanche, la demande de récusation ne pourrait concerner l'ensemble des membres du Conseil constitutionnel. Cela, en effet, constituerait une demande de renvoi pour cause de suspicion légitime et le Conseil, en tant que juridiction ayant un monopole contentieux, ne pourrait pas renvoyer l'affaire devant une autre juridiction ${ }^{82}$. Comme dans le cas précédent, le blocage déboucherait sur un déni de justice, sanctionné par la Cour de Strasbourg qui impose que «les demandes de récusation soient traitées de manière telle qu'elles ne puissent entraîner une paralysie de la justice ou un retard excessif dans l'administration de celle-ci ${ }^{83}$.

\section{B. Les pratiques procédurales du Conseil constitutionnel}

Une fois la demande de récusation enregistrée au secrétariat général, elle est communiquée au membre du Conseil constitutionnel intéressé. Le juge peut décider d'acquiescer à la récusation ou bien de refuser la demande. Lorsque le juge refuse d'accueillir la demande de récusation, l'article 4 prévoit que le Conseil examine la requête, excluant de la décision le membre intéressé. Aucune obligation n'est imposée au Conseil quant aux temps et aux modalités de cet examen. Notamment, sont remis à l'appréciation du Conseil le choix de motiver la décision adoptée (1), et le choix des temps et des modalités de la communication aux requérants de la décision (2).

\section{1) La motivation de la décision}


Avant que la première demande de récusation soit déposée, la doctrine s'interrogeait sur le choix que le Conseil aurait opéré concernant la motivation de la décision de rejet ${ }^{84}$. En effet, à la différence des juridictions administratives ${ }^{85}$, le règlement intérieur n'impose pas de motiver la décision de rejet mais ne l'empêche pas non plus. La réponse du secrétaire général du Conseil du 26 mai 2011, adressée personnellement au requérant, a manifesté la volonté des Sages de ne pas motiver les décisions de rejet, se limitant à affirmer que : «les motifs invoqués à l'appui de la demande de récusation n'étaient pas de nature à faire obstacle à leur [des juges Debré, Haenel et Steinmetz] participation à la décision du Conseil ». Cela soulève un certain nombre de questions.

Tout d'abord, puisqu'il s'agit d'un incident de procédure qui a des conséquences importantes sur le déroulement du contentieux et le délibéré final, la décision concernant les demandes de récusation devrait être considérée comme une véritable décision juridictionnelle et non comme une mesure d'administration interne au Conseil. Dans le cas du déport volontaire, l'absence de toute obligation à la charge du juge de justifier son choix et le manque de tout contrôle de la part du Conseil sur la décision d'abstention ${ }^{86}$ sont motivés par la nécessité de garantir l'autonomie des Sages. La récusation présente en revanche d'autres enjeux. Les parties agissent afin de satisfaire leur droit à une juridiction impartiale. La délibération fait suite à une demande formelle et «spécialement motivée » ${ }^{87}$ et elle est prise par l'organe dans son ensemble afin de donner des garanties plus importantes aux requérants. Il serait donc judicieux que les parties aient droit à une réponse motivée, pour qu'elles sachent pouvoir compter sur une cour impartiale. Cela permettrait d'ailleurs de rendre publique la jurisprudence du Conseil sur les causes de récusation, donnant ainsi aux parties un cadre de référence pour exercer leur droit.

Le choix du Conseil de ne pas motiver ses décisions en matière de récusation peut s'expliquer par la crainte d'une médiatisation excessive des membres, qui aurait par effet une délégitimation de l'organe aux yeux des citoyens et des autres institutions. Il nous semble, au contraire, que le choix de la transparence permettrait de renforcer la légitimité du Conseil tout spécialement dans cette période où, suite à l'introduction de la question prioritaire de constitutionnalité, cet organe accède au plein titre aux fonctions d'une Cour.

L'exemple des juridictions internes et des solutions étrangères pourrait orienter le Conseil vers des pratiques caractérisées par une plus grande transparence. Le juge judiciaire français a en effet décidé, dans le silence du Code de procédure civile, de motiver les décisions de rejet des demandes de récusation 88. Quant au droit étranger, la Cour constitutionnelle belge, sans fondement textuel, statue sur les demandes de récusation par des arrêts motivés ${ }^{89}$. Le Tribunal constitutionnel espagnol répond aux demandes de récusation par des ordonnances motivées avec beaucoup de précision et contenant parfois des opinions dissidentes $^{90}$. L'article 19 de la loi sur la Cour constitutionnelle fédérale allemande du 12 mars 1951 prévoit explicitement que les décisions sur les demandes de récusation adoptées par la Cour en assemblée plénière soient motivées ${ }^{91}$. Et encore, aux États-Unis, la décision des juges de la Cour suprême d'acquiescer ou non à une demande de récusation n'est pas soumis à un contrôle de la part de l'organe collégial. Cependant, le refus est motivé parfois très longuement par le juge visé dans des mémorandums qui accompagnent la décision de conformité constitutionnelle ${ }^{92}$. En Afrique du Sud, le choix de motiver la décision relative à une demande de récusation tient explicitement à l'exigence de légitimer le travail de la Cour aux yeux des citoyens et des autres institutions. Lors de la première demande de récusation en $1998^{93}$, le juge Richard Goldstone expliquant l'affaire, s'exprimait ainsi : «Je crois que la décision de la Cour a été généralement acceptée mais je crois aussi qu'il était nécessaire que nous ayons ce débat public sur une telle question et que nous donnions la pleine justification de notre décision par une motivation 
détaillée de façon inhabituelle ${ }^{94}$.

En conclusion, la motivation de la décision du Conseil au sujet d'une demande de récusation permettrait, d'une part, de garantir la transparence d'un acte qui contribue de façon décisive à préserver l'impartialité et, par conséquent, la légitimité de l'organe ; d'autre part, elle contribuerait à l'émergence d'une jurisprudence capable de donner un cadre de référence aux parties du contentieux et de réduire d'autant la marge d'appréciation du juge.

\section{2) Les temps et les modalités de la communication de la décision}

En l'absence de toute indication réglementaire, le secrétaire général du Conseil a choisi de communiquer au requérant la décision prise par les juges de la rue Montpensier dès son adoption, le 26 mai 2011, c'està-dire neuf jours après l'enregistrement de la demande et plus d'un mois avant l'audience publique, qui s'est tenue le 30 juin 2011.

Le choix du Conseil semble se conformer au principe de transparence de la procédure du contentieux constitutionnel. La garantie de ce principe pourrait d'ailleurs être renforcée au travers de la coordination de la communication du déport volontaire des membres du Conseil avec la décision sur la récusation. L'exercice du droit d'abstention par un juge, en effet, n'est communiqué qu'à l'audience ${ }^{95}$ et il n'est d'ailleurs référencé que dans les commentaires des décisions au Nouveaux cahiers du Conseil constitutionnel ou bien dans les enregistrements vidéo des audiences ${ }^{96}$. Dans les décisions QPC publiées au Journal officiel sont indiqués exclusivement les présents et les absents, sans explicitation des motifs. La communication aux parties d'éventuels déports avant l'audience publique, dans la période où la récusation peut encore être demandée, permettrait d'éviter des récusations hasardées et montrerait le respect porté par le Conseil et ses membres au principe d'impartialité de la juridiction ${ }^{97}$. La seule limite à la coordination des deux mécanismes est constituée par la brièveté du délai imparti pour faire enregistrer la demande de récusation au secrétariat général ${ }^{98}$. Toutefois, puisque la pratique du déport est certainement préférable dans un souci de simplicité et d'image du Conseil, il serait souhaitable de communiquer très rapidement les décisions de déport des juges afin d'anticiper les demandes de récusation, notamment lorsque le risque de partialité subjective ou objective est manifeste.

Quant à la publicité de la décision, lors de la première demande de récusation, les Sages ont préféré de ne pas rendre publiques ni la demande de M. Montebourg, ni la lettre de réponse rédigée par le secrétaire général et contenant le délibéré non motivé du Conseil. Il s'agit d'un choix cohérent avec la volonté de ne pas fournir les raisons qui ont déterminé les juges. Le Conseil conçoit la décision en matière de récusation comme un acte d'administration interne et non pas comme un acte juridictionnel.

Toutefois, l'affaire en question montre les risques d'une pratique non transparente. M. Montebourg a fait publier sa demande et la lettre du secrétaire général sur le blog du quotidien Le Monde, sans aucune autorisation préventive de la part du Conseil ${ }^{99}$. La publication au Journal officiel de ces actes, outre à être gage d'une légitimité renouvelée de l'organe constitutionnel, permettrait d'éviter toute instrumentation de l'affaire par les parties du contentieux. Ces dernières pourraient tenter de faire pression sur le Conseil avant l'audience publique, recourant, comme dans ce premier cas, à la force divulgatrice des médias.

\section{Conclusion}


Les premières demandes de récusation ont répondu à certaines questions posées au niveau théorique avant la mise en application de l'article 4 du règlement, tout en faisant resurgir de nouvelles interrogations.

Tout d'abord, la question des causes de récusation, et notamment de l'interprétation de la limite posée par le règlement, est traitée et les premières règles prétoriennes se dessinent. Le Conseil constitutionnel confirme la légitimité de la cause d'exclusion introduite par l'article 4 et l'applique de façon stricte au cas d'espèce, rejetant la contestation de $\mathrm{M}$. Montebourg quant à la conformité de cette règle à la jurisprudence de la Cour européenne des droits de l'homme. Toutefois, l'analyse des arguments du requérant et des arrêts de la Cour de Strasbourg montre qu'une pleine garantie de l'impartialité du Conseil exigerait qu'un juge ayant participé à l'élaboration d'une loi ne puisse pas statuer ensuite sur sa constitutionnalité. Si pour le Conseil le vote d'une loi ne constitue pas une cause valable de récusation, la jurisprudence européenne semble contredire cette interprétation. Les Sages considèrent que, pour être récusé, un juge doit avoir manifesté par le passé son appréciation sur la constitutionnalité de la norme faisant l'objet du contrôle. Mais le vote en faveur d'une loi par un parlementaire n'indique- t-il pas implicitement qu'il considère la disposition législative conforme au bloc ? Et la saisine manquée du Conseil pour effectuer un contrôle a priori de la loi n'est-elle pas une autre manifestation implicite de l'appréciation de sa constitutionnalité par le parlementaire, ou le président de l'Assemblée Nationale ou bien le Président de la République devenus juges constitutionnels par la suite?

La réponse du Conseil constitutionnel ne permet pas de répondre à ces questions. La lettre adressée à M. Montebourg se limite à rejeter les demandes de récusation de Messieurs Debré, Haenel et Steinmetz, à rassurer les parties sur l'absence chronique de M. Chirac et à faire entendre que Messieurs Charasse et Barrot se seraient de toute façon abstenus. L'absence d'une motivation de la décision des Sages fait obstacle à la création de règles jurisprudentielles capables de limiter, à la fois, les possibles abus des requérants et la marge d'appréciation du Conseil. La réponse du Conseil permet juste de déduire par intuition certains critères interprétatifs, mais non pas d'établir des règles interprétatives constantes.

La réticence du Conseil à être plus transparent dans sa pratique procédurale est liée aux caractéristiques de la récusation, qui se présente comme un mécanisme «à double tranchant ». Si d'une part il semble garantir l'impartialité de la juridiction constitutionnelle, d'autre part il menace sa légitimité. Une utilisation répétée et abusive de cet instrument pourrait en effet conduire à un certain discrédit de l'institution. Cette crainte doit toutefois être nuancée. En effet, les causes de récusation doivent toujours être prouvées par les parties, puisque l'impartialité du juge est présumée. De ce fait, une utilisation abusive de la récusation portera atteinte plus à la crédibilité des parties qu'à la légitimité du Conseil constitutionnel. En conclusion, un organe juridictionnel qui est capable d'admettre la partialité de l'un de ses juges dans une affaire donnée ne ressort pas affaibli de cette procédure, mais renforcé. Aux yeux des citoyens, il garantit en effet l'impartialité de l'exercice de la fonction juridictionnelle, au-delà de tout intérêt de protection corporative. 


\section{Notes de bas de page}

1 Notamment, l'arrêt CEDH, 23 juin 1993, Ruiz-Mateos c/Espagne, Série A, n 262, G. COHENJONATHAN, «L'arrêt Ruiz-Mateos contre Espagne », RFDC, n 17, 1994, pp. 175-183. Suite à cette décision, les garanties contenues dans l'article $6 \$ 1$ ont été étendues aux procédures des juridictions constitutionnelles. La Cour européenne ne fait plus de distinction entre contentieux ordinaire et contentieux constitutionnel. Dès lors qu'une procédure constitutionnelle -recours direct ou question préjudicielle - est étroitement liée à des droits et obligations de caractère civil ou à une accusation en matière pénale, «il convient d'avoir égard, non pas au caractère nécessairement objectif de tout contentieux constitutionnel », mais au caractère subjectif du litige auquel il est rattaché. M. VERDUSSEN, «Le juge constitutionnel face à l'article $6 \S 1$ de la Convention européenne des droits de l'homme », RBCD, 1994, p. 135.

La jurisprudence Ruiz-Mateos c/Espagne a été confirmée par les arrêts : CEDH, arrêt 19 avril 1993, Kraska c/Suisse, (Série A n ${ }^{\circ} 254$ B), § 26 ; arrêt 1er juillet 1997, Pammel c/RFA, (Série A, 1997-IV, n ${ }^{\circ}$ 40, p. 1096) ; arrêt 1er juillet 1997, Probstmeier c/R.F.A., (Série A, 1997-IV, nº 40, p. 1123).

2 Le 13 février 2009, à l'occasion de la visite du président de la CEDH au Conseil constitutionnel, JeanLouis Pezant a affirmé clairement l'adhésion du contentieux constitutionnel a posteriori aux règles du procès équitable : «[...] Nous connaissons votre arrêt du 23 juin 1993, Ruiz-Mateos c/ Espagne, qui fut le coup de tonnerre dans l'existence paisible des cours constitutionnelles chez lesquelles il a d'ailleurs suscité quelques réserves ou quelques interrogations, et nous savons que, désormais, à la suite de la révision de notre Constitution du 23 juillet 2008, nous avons vocation à prendre en compte des règles dont vous avez jugé qu'elles étaient applicables aux instances chargées d'un contrôle de constitutionnalité de la même nature que celui qui va désormais nous incomber ». Cité par P. COSTANZO, La «rtuova » Costituzione délia Francia, Torino, Giappichelli, 2009, p-476.

3 L'origine de ce principe est très ancienne. L'Ancien Testament fait référence à plusieurs reprises à l'impartialité du juge comme à une condition nécessaire pour la réalisation de la Justice sur Terre et l'expression même de la volonté divine. V., B. BERNABE, La récusation des juges, Paris, LGDJ, 2009, pp. 9-10.

4 En 1987, bien avant l'arrêt de référence Ruiz-Mateos , le respect de la garantie d'impartialité avait déjà fait l'objet d'un examen par la CEDH. Arrêt 23 avril 1987, Ettl et autres d Autriche, Série A, n 117, §3335 .

5 G. ZAGREBELSKY, La legge e la sua giustizia, Bologna, Il Mulino, 2008, p. 321.

6 S. GUINCHARD, «Indépendance et impartialité du juge. Les principes de droit fondamental », in J. VAN COMPERNOIXE et G. TARZIA, L'impartialité du juge et de l'arbitre, Bruxelles, Bruylant, 2006, p. 3 .

7 Idem, p. 4.

$8 \mathrm{CEDH}$, 1er octobre 1982, Piersack c/Belgique, série A, n 53, §30. 
9 E. GLASER, concl. sur CE, sect., avis cont., 12 mai 2004, Cne de Rogerville, RFDA, 2004, p. 723.

10 Ibidem. V. également : J. THOMAS, L'indépendance du Conseil constitutionnel, Paris, LGDJ, 2010, p. 10 et pp. $78-86$.

11 V. à ce sujet les analyses de : L. FAVOREU, «La légitimité du Juge constitutionnel », RIDC, vol. 46, 1994, n² 2, p. 557-581; J. THOMAS, L'indépendance du Conseil constitutionnel, op. cit.

12 Décision portant règlement intérieur sur la procédure suivie devant le Conseil constitutionnel pour les questions prioritaires de constitutionnalité du 4 février 2010 (J0 du 18 février 2010, p. 2986).

$13 \S 18$ de la loi relative à la Cour constitutionnelle allemande du 12 mars 1951.

14 Art. 101, loi spéciale sur la Cour constitutionnelle belge du 6 janvier 1989.

15 Art. 80, loi organique 2/1979 du 3 octobre 1979 -

16 Loi organique 28/82 du 15 novembre 1982, relative au Tribunal constitutionnel portugais.

17 L'Italie reste l'un des rares pays européens où les causes d'abstention et de récusation des juges n'ont pas trouvé application dans les jugements devant la Cour constitutionnelle. Pour une analyse comparée des systèmes français et italien v. F. JACQUELOT, «Regards comparés sur l'abstention et les récusations au sein du Conseil constitutionnel et de la Cour constitutionnelle italienne : histoire d'une impartialité reprogrammée », Constitutions, 2011, p. 347 s.

18 La compétence juridique du Conseil à encadrer les deux mécanismes n'apparaît pas immédiatement fondée. En effet, l'art. 56 de l'ordonnance du 7 novembre 1958 restreint l'autonomie normative de l'organe prévoyant que ce dernier puisse compléter par son règlement intérieur seulement les règles de procédure applicables devant lui, édictées par le titre II de ladite ordonnance. Or, les droits de récusation et d'abstention ne rentrent pas dans ce titre, consacré aux différents chefs de compétence du Conseil, mais plutôt dans le titre I, relatif à son organisation. Toutefois, par le passé, le Conseil a déjà poussé son autonomie réglementaire au-delà du cadre strict imposé par l'article 56. Notamment, la décision $n^{\circ} 2001$ 446 DC du 27 juin 2001 portant règlement intérieur sur les archives du Conseil, bien qu'adoptée en vertu de l'article 56, ne concernait pas, à proprement parler, des règles de procédure. La question du fondement juridique de ce règlement a été résolue par l'arrêt Brouant, rendu par le Conseil d'Etat le 25 octobre 2002 (CE, Ass., 25 octobre 2002, Brouant, Rec. 345, concl. Goulard, RFDA , 2003, n 1, pp. 1-8 ; J.-P. CAMBY, «L'autonomie des pouvoirs publics, limite de la compétence du juge administratif (réflexion sur l'arrêt Brouant du Conseil d'État du 25 octobre 2002)», RDP, 2002, pp. 1855-1870 ; L. FAVOREU, «Le Conseil d'État respecte l'indépendance du Conseil constitutionnel », Rec. Dalloz, 2002, n 43, pp. 3287 3289 et «Points de vue sur l'arrêt Brouant », RFDA, 2003, pp. 8-13 ; G. GOULARD, «Conclusions sur Conseil d'État, Assemblée, 25 octobre $2002 »$, RFDA, 2003, n 1, pp. 1-8; O. JOUANJAN et P. GONOD, «Points de vue sur l'arrêt Brouant», RFDA, 2003, n 1, pp. 14-22). Suivant la jurisprudence Brouant, le régime des récusations et des abstentions n'est pas dissociable des conditions dans lesquelles le Conseil constitutionnel poursuit les missions qui lui sont confiées par la Constitution (en ce sens v. aussi arrêt CE $\mathrm{n}^{\circ}$ 258180, 9 novembre 2005, AJDA, 2006, p. 147, obs. F. Donnât ;JCA A, 2006, p. 263, note P. Cassia). La réglementation des deux instruments est étroitement liée à la fonction juridictionnelle du Conseil, et 
notamment à son exercice impartial et indépendant. En tout cas, rien n'empêche que le législateur dans le futur, considérant l'importance de la matière, décide de promulguer une loi organique encadrant les deux mécanismes. Comme pour la réglementation des archives du Conseil constitutionnel (loi organique $\mathrm{n}^{\circ}$ 2008-695 du 15 juillet 2008), la loi organique se substituerait alors à l'article 4 du règlement intérieur de 2010.

19 E. CARTIER, «La récusation et le déport devant le Conseil constitutionnel : cote mal taillée ou réelle avancée ? », LPA, 5 mai 2011, n 89, p. 24.

20 La QPC n ${ }^{\circ} 11-90.025$ soulevée le 8 mars 2011 par le Tribunal de grande instance de Paris (11e chambre correctionnelle) dans le cadre du procès concernant l'ancien président Chirac avait ouvert une première réflexion sur l'exercice du droit de récusation par les parties et ses conséquences pour le Conseil («Le Conseil constitutionnel embarrassé par le cas Chirac », Blog Le Monde, 10 mars 201 1, http ://libertes.blog.lemonde.fr/201 1/03/10/le-conseil-constitutionnel-embarrasse-par-le-cas-chirac/). Le traitement de ces questions fut toutefois renvoyé, puisque la Cour de cassation décida de ne pas saisir le Conseil constitutionnel (Cour cass., ass., arrêt nº 595 du 20 mai 201 1).

21 B. BERNABE, La récusation des juges, op. cit., p. 13.

22 La CEDH a clairement affirmé qu'un «requérant ne saurait prétendre avoir eu des motifs légitimes de douter de l'impartialité du tribunal qui l'a jugé alors qu'il pouvait en récuser la composition mais s'en est abstenu ». Arrêt Bulut c. Autriche, 22 février 1996, § 34.

23 La demande de récusation a été posée relativement aux questions prioritaires de constitutionnalité $n^{\circ}$ 2011-142 et 2011-143 renvoyées par le Conseil d'État au Conseil constitutionnel le 20 avril 2011 (CE, n 346205, lere et 6e sous-sections réunies, Rec. Leb.). Plusieurs départements avaient intenté un recours à l'encontre du refus de l'État de leur attribuer des fonds supplémentaires pour faire face à leurs nouvelles compétences (allocation personnalisée d'autonomie, prestation de compensation du handicap et revenu de solidarité active). Le Conseil constitutionnel a statué sur ces QPC le 30 juin 2011 (v. Commentaire au Nouveaux cab. Cons, const., décis. n 2011-142/145 QPC, 2011-143 QPC).

24 Art. 219 de la loi organique 6/1985 du 1er juillet 1985 relative au pouvoir judiciaire.

25 Art. 828 du Code judiciaire.

26 Art. 18 de la loi sur la Cour constitutionnelle fédérale du 12 mars 1951.

27 Civ. lère, 28 avril 1998, bull. n I, n 155. L. CADIET, «Droit judiciaire privé », JCP G., 21 octobre 1998, n 43 ; B. BERNABE, La récusation des juges, op. cit., pp. 359-362 ; M. GUILLAUME, «Le règlement intérieur sur la procédure suivie devant le Conseil constitutionnel pour les questions prioritaires de constitutionnalité », LPA, $n^{\circ} 38,23$ février 2010, p. 6.

28 Se fondant sur l'article $6 \S 1$ de la Convention européenne des droits de l'homme, la Cour a estimé que «si le domaine de la récusation doit être défini en contemplation du droit au procès équitable, les exigences de ce droit s'imposent aussi à la procédure de récusation ». Civ., 2e, 10 juin 1998, L. CADIET ,JCP G. 1998, IV, 2746, p. 1856. Dans le même sens, en matière de récusation, le Code de justice administrative 
de 2001 ne fait référence qu'à l'existence d'«une raison sérieuse de mettre en doute » l'impartialité du juge, C. just, adm., art. L. 721-1.

29 Art. 4, 4e al., règlement intérieur.

30 F. JACQUELOT, «Regards comparés sur l'abstention et les récusations au sein du Conseil constitutionnel et de la Cour constitutionnelle italienne : histoire d'une impartialité reprogrammée », cit., p. 350 .

31 Demande de récusation du département de Saône-et-Loire, 17 mai 2011, §2.1, publiée sur le site : http ://libertes.blog.lemonde.fr/2011/05/31/premieres-demandes-de-recusation-au-conseil-constitutionnel/, 31 mai 2011.

32 M. Guillaume, cit., p. 6.

33 CEDH, 6 juin 2000, Morel c/France, req. n³4130/96, Rec. 2000-VI.

34 §45. Dans le même sens : CEDH, 24 mai 1989, Hauschildt c/Danemark, Série A n ${ }^{\circ} 154$, p. $22, \S 50$; 24 août 1993, Nortier c/Pays Bas, Série A n 267, p. 15, §33 ; 22 avril 1994, Saraiva de Carvalho c/Portugal, Série A n 286-B, p. 38, §35.

35 M. Guillaume, cit. , p. 6.

36 Arrêt Morel d France, cit., $\$ 45$.

37 CEDH, 8 février 2000, McGonnell c/Royaume-Uni, req. n 28488/95-

38 Selon la Cour européenne, en matière d'impartialité du juge, «même les apparences peuvent revêtir de l'importance »(CEDH, 6 juin 2000, Morel c/France, cit., § 42). CEDH, 17 janvier 1970, Delcourt c/Belgique, série A, $\mathrm{n}^{\circ} 11, \S 31 ; 22$ octobre 1984, Srameck d Autriche, série A, $\mathrm{n}^{\circ} 84 ; 28$ avril 1988, Belilos c/Suisse, série A, $\mathrm{n}^{\circ} 132 ; 7$ juin 2001, Kress c/France, Rec. 2001-VI ; 3 mars 2005, Brudnicka et autres d Pologne, Rec. 2005-11 ; 12 mai 2005, Ocalan c/Turquie, Rec. 2005-IV. Sur la théorie de l'apparence v. : B. BEIGNER et C. BLERY, «L'impartialité du juge, entre apparence et réalité », Rec. Dalloz, 2001, Ch., pp. 2427-2433 ; D. CHABANOL, «Théorie de l'apparence ou apparence de théorie ? Humeurs autour de l'arrêt Kress », AJDA, 2002, Ch., pp. 9-12

$39 \S 53$.

$40 \S 55$.

$41 \S 52$.

$42 \S 54$.

43 CEDH, 28 septembre 1995, Procola c/Luxembourg, série A, n 326 ; Gaz. Pal., 18 nov. 1995, flash, note L. PETTITI ; JD1, 1 996, p. 253, obs. O. de F. ; D., 1996, p. 301, note F. BENOIT-RHOMER ; AJDA, 
1996, p. 383, chron. J.-F. FLAUSS ;JCP, 1996, I, p. 3910, obs. F. SUDRE ; ibid., 1997, I, p. 4017, chron. PETIT J. ; RFDA, 1996, p. 777, note J.-L. AUTIN et F. SUDRE ; RTDH, 1996, p. 275, note D. SPIELMANN ; RUDH, 1996, 1, chron. F. SUDRE.

44 S. GUINCHARD, «Indépendance et impartialité du juge. Les principes de droit fondamental », op. cit., p. 31.

45 Idem, p. 29.

46 Art. 34 de la loi spéciale du 6 janvier 1989 modifiée.

47 Un arrêt récent de la Cour constitutionnelle belge explicite bien la préoccupation que l'exercice du droit de récusation puisse bloquer le fonctionnement de l'organe. Etant donné que la Cour est la seule juridiction compétente pour effectuer un contrôle de constitutionnalité et que sa loi organique ne prévoit pas la possibilité de nommer des juges ad hoc, «l'application des causes de récusation ne peut avoir pour effet que la Cour ne puisse plus délibérer ». Arrêt n 157/2009, B.5, cité par M. BOSSUYT, R. LEYSEN, B. RENAULD, «Séparation des pouvoirs et indépendance des cours constitutionnelles et instances équivalentes. Rapport de la Cour constitutionnelle de Belgique », 2' Congrès de la Conférence mondiale sur la justice constitutionnelle, Rio de Janeiro, 16-18 janvier 2011, pp. 10-11.

48 Déclaration du Garde des Sceaux lors de la présentation du projet de Constitution devant le Conseil d'État. Cité par GENEVOIS (B.), La jurisprudence du Conseil constitutionnel, Paris, Ed. STH, p. 5.

49 D. TURPIN, Contentieux constitutionnel , Paris, PUF, 1986, pp. 207-208.

50 «Il n'y a pas une véritable garantie de la Constitution si ce lien [entre Constitution et politique] est ignoré ou méprisé. L'interprétation la plus abstraite et impolitique de la Constitution pourrait conduire non pas à la plus grande garantie, mais à sa ruine »(ZAGREBELSKY (G.), La legge e la sua giustizia, op. cit., p. 299). V. aussi : J.-M. DENQUIN, «Justice constitutionnelle et justice politique », in C. GREWE, O. JOUANJAN [et al.] (dit. par), La notion de "justice constitutionnelle", Paris, Dalloz, 2005, p. 75.

51 En ce sens : P. WACHSMANN, «Sur la composition du Conseil constitutionnel », Jus Politicum, $\mathrm{n}^{\circ}$ 5, 2010, pp. 14-16.

52 Demande de récusation présentée par le Département de Saône-et-Loire, cit., p. 8. Sur l'applicabilité directe de la Convention EDH et ses conséquences, F. SUDRE, Droit international et européen des droits de l'homme, Paris, PUF, 1999, p. 143 et 184.

53 Les six juges sont récusés dans le cadre de la QPC n 2011-142 concernant l'article 4 de la loi du 18 décembre 2003 et de l'article 59 de la loi du 30 décembre 2003. S'agissant des articles 11 et 12 de la loi $\mathrm{n}^{\circ}$ 2004-626 du 30 juin 2004, des articles L. 14-10-5 et L. 14-10-6 du Code de l'action sociale et des familles (QPC $n^{\circ} 2011-143$ ) et de l'article 2 de la loi $n^{\circ} 2005-1720$ du 30 décembre 2005 (QPC n $^{\circ} 2011$ 142), M. Montebourg demande la récusation de M. Charasse, de M. Haenel, de M. Debré et de M. Chirac. Enfin, concernant l'article L. 262-24 du Code de l'action sociale et des familles (QPC n $\left.{ }^{\circ} 2011-142\right)$ le président du département Saône-et-Loire demande la récusation de M. Charasse et de M. Haenel. 
54 Demande de récusation, cit., p. 7.

$55 \S 52$, McGonnell c ! Royaume-Uni, cit.

56 Demande de récusation, cit., p. 7.

57 Loi n 62-1292 du 6 novembre 1962, J0 du 7 novembre 1962, p. 10762.

58 Loi n $^{\circ}$ 2000-964 du 2 octobre 2000, J0 du 3 octobre 2000, p. 15582.

59 Art. 5, Const.

60 P. WACHSMANN, cit., p. 26.

61 Selon Patrick Wachsmann, étant donné le rôle politique actif du président, ce dernier «n'est plus véritablement en position de se prononcer impartialement sur la constitutionnalité des lois, mêmes nouvelles ». Ibid.

62 Selon M. Marc Guillaume «le seul fait d'avoir participé à l'élaboration de la norme contestée ne constitue pas en soi une cause de récusation ». Cependant, «il ne devrait en aller autrement que si les actes accomplis impliquent que leur auteur a porté une appréciation sur la constitutionnalité de cette norme » (cit. , p. 6).

63 Selon l'expression utilisée par : G. ZAGREBELSKY, La legge e la sua giustizia, op. cit., p. 124.

64 Demande de récusation, cit., p. 7.

65 La lettre de réponse a été publiée sur le site : http ://libertes.blog.lemonde.fr/2011/05/31/premieresdemandes-de-recusation-au-conseil-constitutionnel/.

66 Sur la motivation de la décision du Conseil v. infra.

67 Commentaire au Nouveaux Cahiers du Conseil constitutionnel sur la décision n 201 1-143/144 QPC du 30 juin 2011, cit.. Pour la décision n ${ }^{\circ}$ 2011-142/145 QPC du 30 juin, le commentaire se limite à affirmer que «MM. Michel Charasse et Jacques Barrot n'ont pas siégé dans ces QPC », cit.

68 L'art. 4, 1er al., du règlement se limite à préciser que «tout membre du Conseil constitutionnel qui estime devoir s'abstenir de siéger en informe le président ». Ainsi, le juge qui décide de s'abstenir prend sa décision en totale autonomie, sans être soumis à aucun contrôle de la part du Conseil. Le membre communique sa volonté au président, sans devoir exposer publiquement les motifs de son choix. Sur ce point v. P. BON (P.), cit., p. 2012.

69 E. Cartier, cit., pp. 24-25.

70 II s'agit de l'art. 123 de la loi du 13 août 2004 sur les fusions de communes (QPC n 2010-12), et des dispositions de la loi de finances rectificative du 30 décembre 2008 relatives à la compensation partielle 
des charges induites par le traitement des demandes de CNI et de passeport par les maires $\left(\mathrm{QPC}^{\circ}{ }^{2010-}\right.$ 29/37).

71 Notamment, M. Jean-Louis Debré a exercé son droit d'abstention concernant l'article 1er de la loi du 4 mars 2002 dite anti-Perruche, puisque, comme présumé par Emmanuel Cartier (cit., p. 24), il avait été cosignataire d'une proposition de loi à l'origine du dispositif législatif en cause.

72 M. Guillaume, cit. , p. 6.

73 M. BOSSUYT, R. LEYSEN, B. RENAULD, «Séparation des pouvoirs et indépendance des cours constitutionnelles et instances équivalentes. Rapport de la Cour constitutionnelle de Belgique », cit., p. 10. Pour un aperçu des six arrêts rendus en la matière par la Cour constitutionnelle belge depuis 1987 v. le Rapport annuel pour 2009 de la Cour constitutionnelle Belge, p. 13.

74 Art. 1er, al. 3 du règlement intérieur.

75 II s'agit notamment des délais pour déposer des observations en réponse aux premières observations (art. 1er, al. 4), pour faire des observations suite à une audition requise par le Conseil constitutionnel (art. 6), ou encore pour des observations en cas de griefs soulevés d'office (art. 7).

76 F. JACQUELOT (F.), «La procédure de la question prioritaire de constitutionnalité devant le Conseil constitutionnel », AJDA, 10 mai 2010, p. 953.

77 M. Guillaume (M.), cit., p. 4.

78 Art. 4, règlement intérieur.

79 CEDH, 23 juin 1981, Le Compte, Van Leuven et de Meyere d Belgique, série A, n 43, §58; 6 juin 2000, Morel c/France, req. $n^{\circ}$ 34130/96, Rec. 2000-VI, § 41.

80 J. THOMAS (J.), L'indépendance du Conseil constitutionnel, op. cit., p. 111.

81 E. Cartier (E.), cit. , p. 29.

82 Idem, p. 26.

83 CEDH, 22 septembre 1994, Debled c/Belgique, série A, n² 292-B, §37 ; CEDH, 10 juin 1996, Thomann c/Suisse, Recueil 1996-111, p. 815, § 36 ; CEDH, 12 décembre 2002, Sofianopoulos c/Grèce, Req. ${ }^{\circ}$ 1988/02, 1997/02 et 1977/02. Dans le respect de la jurisprudence européenne, la Cour constitutionnelle belge, se prononçant sur la demande de récusation de cinq juges dans la même affaire, a observé qu'elle est la seule juridiction compétente pour effectuer un contrôle de constitutionnalité et que sa loi organique ne prévoit pas la possibilité de nommer des juges ad hoc. Par conséquent, «l'application des causes de récusation ne peut avoir pour effet que la Cour ne puisse plus délibérer ». Arrêt n 157/2009, B.5, cit.

84 E. Cartier, cit. , p. 28. 
85 L'art. R. 721-9 du Code de justice administrative prévoit expressément que «la juridiction, par une décision non motivée, se prononce sur la demande ».

86 Le juge qui décide de s'abstenir communique tout simplement sa volonté au président du Conseil, sans devoir exposer publiquement les motifs de son choix (art. 4, règlement intérieur). Le Conseil semble considérer l'abstention comme une action relevant du seul for intérieur du juge, presque déconnectée de l'organe collégial auquel il appartient. La doctrine n'a pas manqué de critiquer ce choix, soulignant que : «L'abstention n'est pas un privilège personnel du juge constitutionnel mais un devoir destiné à garantir l'impartialité de la juridiction à laquelle il appartient de telle sorte qu'il est normal que cette dernière ait son mot à dire en décidant en dernière instance si, effectivement, la cause d'abstention alléguée par le juge est effectivement fondée ». BON (P.), cit., p. 2012

87 Art. 4, règlement intérieur.

88 B. BERNABE, «Récusation, Abstention »,JCP G, fasc. 685-2010, p. 16.

89 Pour un aperçu des arrêts rendus depuis 1987 par la Cour constitutionnelle belge suite à des demandes de récusation, M. BOSSUYT, R. LEYSEN, B. RENAL ! ! ), «Séparation des pouvoirs et indépendance des cours constitutionnelles et instances équivalentes. Rapport de la Cour constitutionnelle de Belgique», cit., pp. 10-11; et le Rapport annuel pour 2009 de la Cour constitutionnelle Belge, p. 13

90 C'est le cas, par exemple, de l'ordonnance 26/2007 du 5 février 2007 signée par onze juges, c'est-à-dire les douze membres du Tribunal constitutionnel moins le juge visé, et suivie de cinq opinions séparées. Pour un aperçu de la jurisprudence du Tribunal constitutionnel en la matière, P. BON, cit., pp. 2010-2011 ; M. d. C. CALVO SANCHEZ, Control de la imparcialidad del Tribunal constitucional, Atelier, 2009-

91 M. FROMONT, «Dossier : Allemagne », Cah. Cons, const., n 15, janvier 2004 ; «Chronique », AIJC 2001, p. 423 ; AIJC 2000, p. 515 ; AIJC 1999, p. 380 ; A1JC 1998, p. 560 ; AIJC, 1995, XI, p. 958.

92 V., parmi d'autres, le mémorandum du juge Scalia accompagnant l'arrêt Richard B. Cheney, Vice President of the United States, et al. v, United States District Court for the District of Columbia et al., $\mathrm{n}^{\circ}$ 03-475, 18 mars 2004. Il s'agit d'un mémorandum de 21 pages, dans lequel les raisons du juge sont amplement détaillées.

93 Décision SARFU, President of the Republic of South Africa and Others v South African Rugby Football Union and Others (CCT 16/98).

94 A. Chaskalson, X. Philippe, P. Langa, Y. Mogkoro, R. Goldstone, «Dossier : Afrique du sud », Cahiers du Conseil constitutionnel, $\mathrm{n}^{\circ}$ 9, février 2001.

95 À l'occasion des décisions nos 2010-61 (J0 13 novembre 2010, p. 20238) et 2010-29/37 \{JO 23 septembre 2010, p. 17293) QPC, Jean-Louis Debré et Michel Charasse se sont déportés tout en assistant à l'audience et s'abstenant de siéger au délibéré. Le fait d'assister à l'audience porte atteinte à l'apparence d'impartialité de l'organe juridictionnel devant les parties. La pratique devant les juridictions ordinaires en donne confirmation. Devant le Conseil d'Etat, en début de séance, le président annonce les déports et le nom des magistrats remplaçants. Le magistrat qui se déporte quitte ensuite la salle des séances le temps 
de l'affaire. Le déport est donc rendu public et il peut être constaté par les parties. Il serait souhaitable que cette pratique soit adoptée également par les membres du Conseil constitutionnel. En ce sens v. E. CARTIER, cit., p. 25.

96 Une exception à cette règle non écrite est constituée par les absences de Messieurs Barrot et Charasse dans le cadre des QPC n 2011-144 et 2011-145. Dans ces cas, il faut présumer que les deux juges aient résolu de se déporter volontairement (v. supra). Par la lettre du 26 mai 2011, le Conseil a donc annoncé ces déports bien avant l'audience publique.

97 En ce sens également E. CARTIER, cit., p. 29 ; P. BON, cit., p. 2012.

98 V. supra. Devant le juge ordinaire, au contraire, la demande de récusation peut intervenir jusqu'à «la clôture des débats » (Code just, adm., art. R. 721-2 ; CPC, art. 342). Le Conseil d'Etat a même reconnu la possibilité de poser cette demande au-delà de cette limite (CE, sect., 12 octobre 2009, n 311641, Petit).

99 «Premières demandes de récusation au Conseil constitutionnel », Blog Le Monde, http ://libertes.blog.lemonde.fr/2011/05/31/premieres-demandes-de-recusation-au-conseil-constitutionnel/. 